\title{
Quantum-Well-Induced Giant Spin-Orbit Splitting
}

\author{
S. Mathias, ${ }^{1,2, *}$ A. Ruffing, ${ }^{1}$ F. Deicke, ${ }^{1}$ M. Wiesenmayer, ${ }^{3}$ I. Sakar, ${ }^{1}$ G. Bihlmayer, ${ }^{4}$ E. V. Chulkov, ${ }^{5}$ Yu. M. Koroteev, ${ }^{6}$ \\ P. M. Echenique, ${ }^{5}$ M. Bauer, ${ }^{3}$ and M. Aeschlimann ${ }^{1}$ \\ ${ }^{1}$ Department of Physics and Research Center OPTIMAS, University of Kaiserslautern, 67663 Kaiserslautern, Germany \\ ${ }^{2}$ JILA, University of Colorado and National Institute of Standards and Technology, Boulder, Colorado 80309-0440, USA \\ ${ }^{3}$ Institut für Experimentelle und Angewandte Physik, Universität Kiel, 24098 Kiel, Germany \\ ${ }^{4}$ Institut für Festkörperforschung and Institute for Advanced Simulation, Forschungszentrum Jülich, D-52425 Jülich, Germany \\ ${ }^{5}$ Donostia International Physics Center (DIPC), and CFM, Centro Mixto CSIC-UPV/EHU, Departamento de Física de Materiales, \\ UPV/EHU, Apartado 1072, 20080 San Sebastián, Spain \\ ${ }^{6}$ Institute of Strength Physics and Materials Science, RAS, 634021, Tomsk, Russia
}

(Received 30 September 2009; published 8 February 2010)

\begin{abstract}
We report on the observation of a giant spin-orbit splitting of quantum-well states in the unoccupied electronic structure of a $\mathrm{Bi}$ monolayer on $\mathrm{Cu}(111)$. Up to now, Rashba-type splittings of this size have been reported exclusively for surface states in a partial band gap. With these quantum-well states we have experimentally identified a second class of states that show a huge spin-orbit splitting. First-principles electronic structure calculations show that the origin of the spin-orbit splitting is due to the perpendicular potential at the surface and interface of the ultrathin Bi film. This finding allows for the direct possibility to tailor spin-orbit splitting by means of thin-film nanofabrication.
\end{abstract}

PACS numbers: 73.21.Fg, 71.70.Ej, 79.60.Dp

The Rashba-Bychkov effect [1] in a two-dimensional electron gas, which originates from spin-orbit interaction and an asymmetric confinement of the electron gas, can produce spin-split energy bands in nonmagnetic materials without the need to apply any external magnetic field. Consequently, this effect plays a crucial role for various fields and applications in the area of spintronics. In recent years, surface states of $\mathrm{Au}$ [2-5], Bi [6-10], and $\mathrm{Sb}$ [11] were reported to show Rashba splittings at least as large as for bands in conventional semiconductor devices. Recently, Ast et al. [12] identified a new class of materials, long-range ordered surface alloys between heavy elements such as $\mathrm{Bi}$ or $\mathrm{Pb}$ and the light noble metals $\mathrm{Ag}$ and $\mathrm{Cu}$, which show exceptional large spin splittings of its surface states. The intermixing of the different materials and the corresponding in-plane potential variations are responsible for the giant spin splitting here. These alloys are an ideal model Rashba system to study geometrical and topological changes in the Fermi surface of these surface states [1317]. Even more importantly, the Rashba energies can be tuned by changing the composition parameters of the surface alloys $[18,19]$.

A similar class of electronic states evolves by the confinement of electrons in ultrathin metal films. When the thickness of a film is reduced to values comparable to or smaller than the electron coherence length, the electronic band structure evolves into a quantized electron spectrum in the direction perpendicular to the surface [20]. The quantization arises from the standing electron wave pattern supported by the film and depends critically on the film thickness. This has been shown to influence a variety of other physical properties showing so-called quantum-size effects, e.g., [21-24].
In terms of applications and new materials using Rashba split states for spintronic devices, tunable quantum-well (QW) systems seem very attractive, since both the asymmetric confinement by the surface potential and the interface potential should influence the spin-orbit splitting of the electronic states. However, a giant spin-orbit splitting as for the alloy systems cannot be expected, since the quantum-well overlayer atoms are not intermixed with the substrate. Indeed, until now none $[7,25,26]$ or only a very weak splitting of QW states could be found [27], where the energy separation between the bands is even smaller than the intrinsic line width. This has been attributed to the idea that the charge density is located too far from the surface or interface to experience the potential gradient $[7,26]$. Alternatively, it has been claimed that QW states are standing waves and should therefore show no Rashba splitting [28], or that the net effect of competing effects at both surface and interface reduce the spin-orbit splitting in QW systems [27].

In contrast, in our Letter we show the first system with quantum-well induced giant spin-orbit splitting of QW states (Rashba parameters between $\alpha_{R}=1.5 \mathrm{eV} \AA$ and $2.5 \mathrm{eV} \AA$ ). We carried out our investigations using angleresolved two-photon photoemission to access the unoccupied electronic band structure of a Bi monolayer film on $\mathrm{Cu}(111)$. Our findings are fully confirmed by firstprinciples electronic structure calculations. Up to now, Rashba-type splittings of this size have not been reported for electronic states other than surface states in a partial band gap. With these QW states we have found a second class of states which show a remarkably strong Rashba spin-orbit splitting, and which can easily be modified by thin-film engineering. We show that the localization of the 
QW wave function with respect to the perpendicular potential gradient at the film-substrate and film-vacuum interfaces manifests itself in the amount of spin-orbit splitting of the QW states.

Our density-functional theory calculations were performed in the local density approximation, employing the full-potential linearized augmented plane wave method as implemented in the FLEUR code [29]. The surface was simulated by a 16 layer $\mathrm{Cu}$ film, covered with $\mathrm{Bi}$ on one side. The structural model and the choice of the axes was taken from the experiment [30] such that $x$ is taken to be the long axis of the unit cell [Fig. 1(d)]. In the selfconsistent calculations $16 \mathbf{k}_{\|}$-points and about 73 basisfunctions per atom were used and spin-orbit coupling was included as described by Li et al. [31].

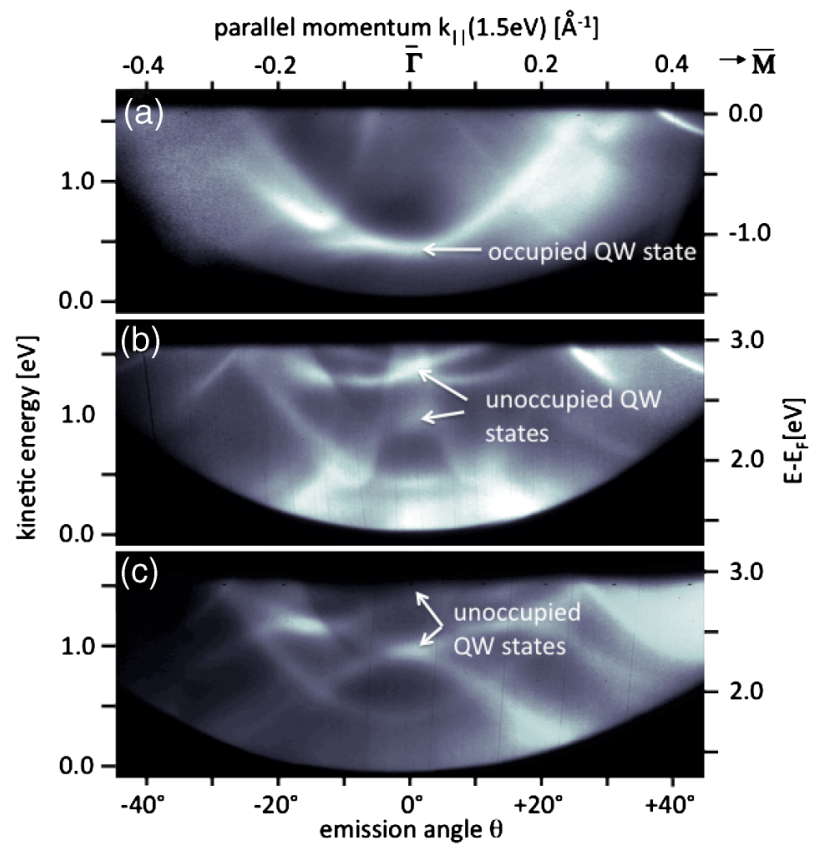

(d)

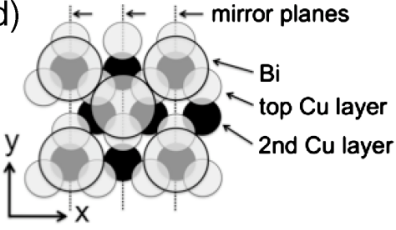

(e)

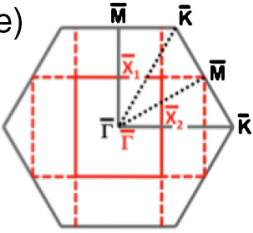

FIG. 1 (color online). (a) 1PPE map and (b),(c) 2PPE maps of the commensurate (a),(b) and incommensurate (c) Bi monolayer on $\mathrm{Cu}(111)$. For generation of the full angle distribution from about $-45^{\circ}$ to $+45^{\circ}$ a tilting of the sample with respect to analyzer and laser light source is required. This results in a modification of the effective polarization of the laser light impinging on the sample surface and leads to the strong asymmetry in the photoemission spectra. The band bottom of the occupied QW state and the crossings of the spin-orbit split unoccupied QW states are marked with white arrows. (d) Schematic of the [2012] $\mathrm{Bi}$ monolayer on $\mathrm{Cu}(111)$. (e) Brillouin zone scheme for the Bi monolayer (red or gray) on $\mathrm{Cu}(111)$ (black).
The angle-resolved one-photon-photoemission (1PPE) and two-photon-photoemission (2PPE) studies were conducted using a hemispherical energy analyzer (SPECS Phoibos 150). The total energy resolution of the analyzer at the pass energy of $20 \mathrm{eV}$ used in these experiments is $20 \mathrm{meV}$, with an angle resolution of $0.3^{\circ}$.

The laser system used for the 1PPE and 2PPE experiments, respectively, is a mode-locked Ti:sapphire laser at $840 \mathrm{~nm}, 82 \mathrm{MHz}$ repetition rate, an energy of $15 \mathrm{~nJ} /$ pulse, and $100 \mathrm{fs}$ pulse width. The output of the Ti:sapphire laser is frequency doubled in 0.2 -mm-thick beta barium borate crystals to produce pulses of $h \nu=2.95 \mathrm{eV}$ and $h \nu=$ $5.9 \mathrm{eV}$. The work function of the investigated surface is 4.40(1) eV and allows additionally to normal photoemission, 1PPE, for 2PPE within a two step excitation process permitting the direct spectroscopic access to intermediate excited states between Fermi edge and vacuum level. For the evaporation of $\mathrm{Bi}$ an effusion cell has been used. $\mathrm{Bi}$ exhibits three different phases on $\mathrm{Cu}(111)$ [30]. For a coverage of $1 / 3$ compared to the substrate a surface alloy is formed with a $(\sqrt{3} \times \sqrt{3}) R 30^{\circ}$ unit cell. The monolayer structures investigated in this work form for increasing coverage to 0.5 . Here, a dealloying occurs, leading to a structure in which the $\mathrm{Bi}$ atoms form zigzag chains. The surface contains three domains of this $\left(\begin{array}{ll}2 & 0 \\ 1 & 2\end{array}\right)$ phase. Finally, at a slightly higher coverage of 0.53 , the $\left(\begin{array}{ll}2 & 0 \\ 1 & 2\end{array}\right)$ unit cell is compressed in one direction, leading to a uniaxialincommensurate monolayer with three rotational domains. Figure 1 shows photoemission spectra of the commensurate (a), (b) and incommensurate (c) $\left(\begin{array}{ll}2 & 0 \\ 1 & 2\end{array}\right)$ monolayer of the $\mathrm{Bi}$ on $\mathrm{Cu}(111) \mathrm{QW}$ system. The photoemission maps show photoemission intensity as a function of kinetic energy $E$ and parallel momentum $k_{\|}$. Spectrum (a) is measured with 1PPE $(h \nu=5.9 \mathrm{eV})$, therefore showing the occupied electronic structure from below the Fermi-level. One can observe a dispersive QW state around the $\bar{\Gamma}$ point at an energy of $E-E_{F}=-1.14(1) \mathrm{eV}$. Photoemission map (b) shows the same structure investigated with 2PPE with $h \nu=$ $2.95 \mathrm{eV}$. The occupied electronic structure is still visible but with much lower intensity. All additional electronic structure originates from intermediate states in the unoccupied regime between Fermi and vacuum level. The dispersive QW state in the unoccupied regime at an energy of about $E-E_{F}=2.76(1) \mathrm{eV}$ is clearly spin-orbit split. A second spin split QW state can be observed at $E-E_{F}=$ 2.33(1) $\mathrm{eV}$, however much more pronounced in the photoemission map of the incommensurate monolayer phase of $\mathrm{Bi}$ on $\mathrm{Cu}(111)$ [Fig. 1(c)].

To determine the nature of the wave functions responsible for the observed spectral features and to elucidate the origin of the large Rashba-type spin-orbit splittings, we performed density-functional theory calculations as described above. Because of the backfolding of the $\mathrm{Cu}$ band structure, the Bi states are observed on a continuum of $\mathrm{Cu}$-derived states (no $L$ band gap, Fig. 2). Also, three 
QW states can clearly be observed: In the occupied region, at $E-E_{F}=-1.0 \mathrm{eV}$ at the $\bar{\Gamma}$ point (blue dots), a $p_{y}$-type state can be identified that is almost dispersionless in $\bar{\Gamma}-\bar{X}_{1}$ direction, while in $\bar{\Gamma}-\bar{X}_{2}$ direction it shows a positive dispersion and a small Rashba-type spin-orbit splitting. This Bi state can be identified with the feature seen at $E-$ $E_{F}=-1.14 \mathrm{eV}$ in the 1PPE map of Fig. 1(a). Notice, that the $\bar{\Gamma}-\bar{M}(\bar{K})$ direction of the clean $\mathrm{Cu}$ substrate corresponds to two possible directions in the Bi monolayer system: one pointing along a vector $\bar{\Gamma}-\bar{X}_{1}\left(\bar{X}_{2}\right)$ and one $60^{\circ}$ rotated to this line [c.f. Brillouin zones shown in Fig. 1(e)].

In the unoccupied region we observe-in nice agreement with the 2PPE spectra-near the zone center two Bi states at $E-E_{F}=2.1 \mathrm{eV}$ (experimental: $2.33 \mathrm{eV}$ ) and $E-E_{F}=2.7 \mathrm{eV}$ (experimental: $2.76 \mathrm{eV}$ ) with pronounced spin-orbit splitting (red dots).

Figure 3 shows a direct comparison of experimental and theoretical data sets in experimental $\bar{\Gamma}-\bar{M}$ and $\bar{\Gamma}-\bar{K}$ directions with the Bi calculated $\bar{\Gamma}-\bar{X}_{1}$ and $\bar{\Gamma}-\bar{X}_{2}$ directions, respectively. The Rashba parameter $\alpha_{R}$ can be determined from the splitting $k_{0}$ and from the effective mass $m^{*}: \alpha_{R}=$ $\hbar^{2} k_{0} / m^{*}$. The Rashba energy $E_{R}$ is defined as $E_{R}=$ $\hbar^{2} k_{0}^{2} / 2 m^{*}$. Experimentally, we evaluated $\alpha_{R}$ from half the slope of the energy spacing versus in-plane momentum. For the upper state around $E-E_{F}=2.7 \mathrm{eV}$ we observe Rashba parameters of $1.63(5) \mathrm{eV} \AA$ in $\bar{\Gamma}-\bar{M}$ and $1.47(5) \mathrm{eV} \AA$ in $\bar{\Gamma}-\bar{K}$ direction. This is in good agreement with the theoretically observed values of $1.8 \mathrm{eV} \AA$ in $\bar{\Gamma}-\bar{X}_{1}$
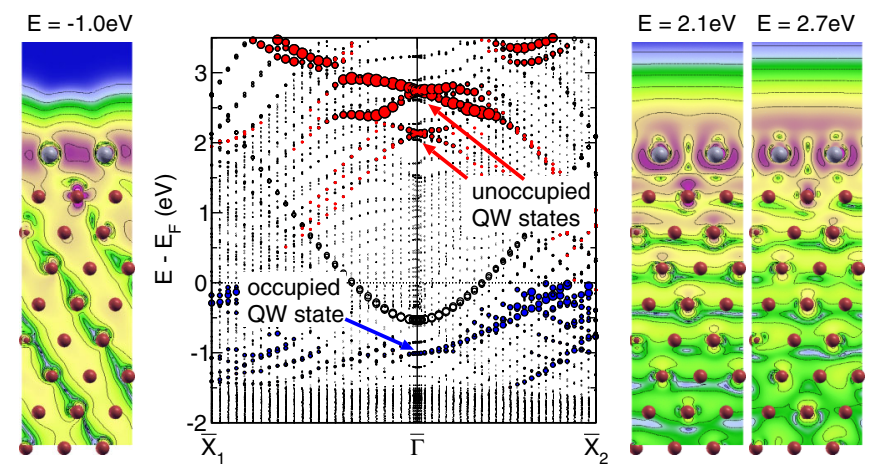

FIG. 2 (color online). Calculated band structure for commensurate monolayer of $\mathrm{Bi}$ on $\mathrm{Cu}(111)$. States marked in red (or gray) and blue (or dark gray) are Bi-derived unoccupied and occupied QW states, $\mathrm{Cu}$ states are shown in black. The size of the symbol is a measure of the weight of the states in the vacuum. For better visibility, the weight of the occupied states (blue) is multiplied by a factor 5 . Cuts through the charge densities of three $\mathrm{Bi}$ states from the $\bar{\Gamma}$ point are shown: at the left, the (occupied) state at $E-E_{F}=-1.0 \mathrm{eV}$ and, at the right, two unoccupied states at $E-E_{F}=2.1$ and $2.7 \mathrm{eV}$. With increasing energy the weight of the states in the vacuum can be seen to increase. The cuts show the upper eight $\mathrm{Cu}$ layers and go through the two $\mathrm{Bi}$ atoms in the unit cell. In the band structure, the $\mathrm{Cu}$ surface state from the other side of the film is visible as black circles. direction and $1.2 \mathrm{eV} \AA$ in $\bar{\Gamma}-\bar{X}_{2}$ direction. Note once again that the $\bar{\Gamma}-\bar{M}$ and $\bar{\Gamma}-\bar{K}$ directions in the experimental data always contain two possible directions in the Bi monolayer system, therefore showing a certain intermediate between the possible directions. If we average the theoretically calculated Rashba-constants over all 3 domains, we get 2.16 and $1.73 \mathrm{eV} \AA$ for $\bar{\Gamma}-\bar{M}$ and $\bar{\Gamma}-\bar{K}$ directions, respectively. We also extracted the Rashba parameters of the lower spin-orbit split QW state around $E-E_{F}=2.3 \mathrm{eV}$, however, for the incommensurate monolayer, where this state is much more pronounced. We find Rashba values of around 2.48(5) $\mathrm{eV} \AA \AA$ in both directions.

The observed giant spin-splitting of our QW states is in the same order of magnitude as the prominent giant spin orbit splitting found for the surface alloy system $\mathrm{Bi} / \mathrm{Ag}(111)$ with $3.05 \mathrm{eV} \AA$ [12]. The respective values are 0.33 and $0.07 \mathrm{eV} \AA$ for the $\mathrm{Au}(111)$ surface state [3] and semiconductor heterostructures [32]. The value recently found for QW states of $\mathrm{Pb}$ on $\mathrm{Si}(111)$ is $0.04 \mathrm{eV} \AA$ [27]. Our experimental observations therefore clearly identify a second type of class, QW states, exhibiting giant spin-orbit split electron states. When we analyze the extension of the wave functions, we find that the weight of the states in the vacuum region increases with increasing energy (size of the symbols in Fig. 2). From an orbital decomposition of these states we infer that they are all of $p_{y}$ character. Therefore, the increasing delocalization towards the vacuum can be interpreted as an effective lowering of the vacuum barrier for the higher-lying states. As we can see from the band structure, this has rigorous consequences for the size of the Rashba-type splitting: while the occupied state at $-1.0 \mathrm{eV}$ is mainly localized inside the Bi layer and is to a large extent ignorant to the

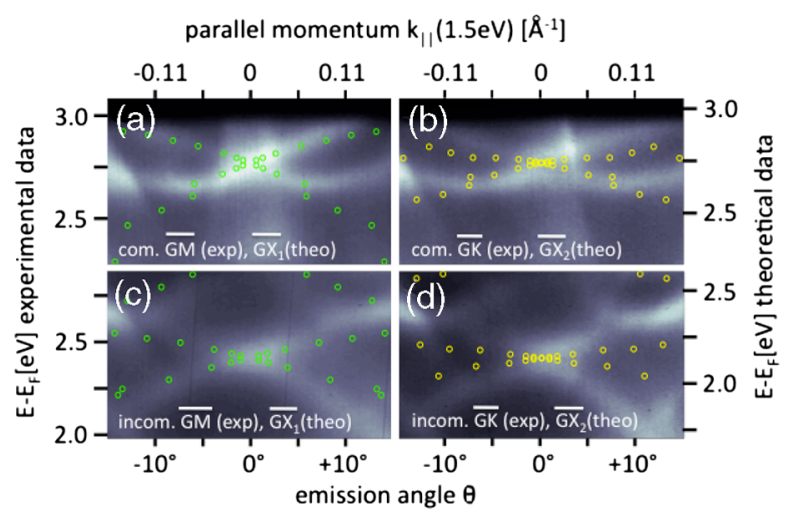

FIG. 3 (color online). Direct comparison of experimental and theoretical data sets. (a) and (b) show the spin split QW state in the commensurate structure around $2.7 \mathrm{eV}$ in $\bar{\Gamma}-\bar{M}$ and $\bar{\Gamma}-\bar{K}$ directions with the calculated $\bar{\Gamma}-\bar{X}_{1}$ and $\bar{\Gamma}-\bar{X}_{2}$ directions, respectively. (c) and (d) show the spin split QW state around $2.1 \mathrm{eV}$ in the incommensurate structure with the according calculated data. Note that the $\bar{\Gamma}-\bar{K}$ and $\bar{\Gamma}-\bar{M}$ directions of the clean $\mathrm{Cu}$ substrate correspond to two possible directions in the Bi overlayer system. 
potential difference between the film/substrate and film/ vacuum interface, the unoccupied states probe with their wave functions this difference and the induced asymmetry of the wave function-shape manifests itself in the much larger spin splitting.

From the geometrical structure of the Bi monolayer on $\mathrm{Cu}(111)$ it can be expected that in-plane potential gradients play a certain role: the $\mathrm{Bi}$ atoms sit on top of a $\mathrm{Cu}$ triangle, slightly displaced in the $y$ direction [Fig. 1(d)]. Since there is a mirror plane perpendicular to the $x$ axis, a potential gradient can only occur along the $y$ direction. From an analysis of the potential at the Bi site, we conclude that there is indeed a gradient $\partial V(\mathbf{r}) / \partial y$ which is similar in size to $\partial V(\mathbf{r}) / \partial z$. But one has to keep in mind that there are two Bi atoms in the unit cell, with potential gradients that are almost equal, but along $y$ they are of opposite sign. A small imbalance of these gradients results from the $\mathrm{Cu}$-subsurface layer: with respect to this layer the two Bi atoms sit (almost) on fcc or hcp sites, respectively. Since the Bi-wave functions extend over both atoms, the influence of the in-plane gradient almost cancels. A signature of this gradient can be found in the occupied Bi state at $-1 \mathrm{eV}$, where a substantial out-of-plane spinpolarization is observed. It is reasonable that this state, which is most localized in the surface plane, shows also the highest sensitivity towards the symmetry breaking in the plane. As expected, this effect is only visible for electrons traveling perpendicular to the gradient, i.e., in $\bar{\Gamma}-\bar{X}_{2}$ direction.

In contrast, the effect on the unoccupied states at 2.1 and $2.7 \mathrm{eV}$ is small. Even if we break the symmetry additionally in our calculations, there is no enhancement of the Rashba-type splitting by the in-plane gradient (not shown), as was proposed by Premper et al. [33]. On the opposite, we see that the Rashba parameter in $\bar{\Gamma}-\bar{X}_{1}$ is larger than in $\bar{\Gamma}-\bar{X}_{2}$ direction, where the electrons should experience the in-plane potential gradient.

Therefore, the origin of the large Rashba parameter in this QW system lies in the out-of-plane gradient $\partial V(r) / \partial z$. Note, that Rashba parameters of this size have also been observed for other systems, e.g., on $\mathrm{Bi}(110)$ [34] or Bi on $\mathrm{Si}$ [35]. But in the former case, this effect was seen for a $p_{z}$-type surface state, and in the latter case by in-plane symmetry reduction of the Bi trimer phase on $\mathrm{Si}$.

In our Letter we show a giant spin splitting for QW states. Our findings are fully confirmed by first-principles electronic structure calculations. The effect is brought by the perpendicular potential gradient to the film, which also confines the electrons in the ultrathin metal film. In contrast to surface electronic systems, bulklike QW systems are much more attractive for possible spintronic applica- tions. They are by far less sensitive to surface perturbations in real systems (adsorbates, defects, strain, etc.) and can be built up to useful devices by sandwich multi-quantum-well heterostructures. Furthermore, properties as binding energy, effective mass and now spin-orbit splitting can easily altered by changing the metal layer thickness, selection of materials or surface and interface engineering.

This work was supported by the DFG GRK 792 and the DFG SFB/TRR49, the UPV/EHU (Grant No. GIC07IT36607), the Departamento de Educación del Gobierno Vasco, and the Spanish MCyT (Grant No. FIS200766711C0101).

*Corresponding author. smathias@jila.colorado.edu

[1] Y. A. Bychkov et al., JETP Lett. 39, 78 (1984).

[2] I. Barke et al., Phys. Rev. Lett. 97, 226405 (2006).

[3] M. Hoesch et al., Phys. Rev. B 69, 241401(R) (2004).

[4] S. LaShell, B. A. McDougall, and E. Jensen, Phys. Rev. Lett. 77, 3419 (1996).

[5] G. Nicolay et al., Phys. Rev. B 65, 033407 (2001).

[6] Y. Koroteev et al., Phys. Rev. Lett. 93, 046403 (2004).

[7] T. Hirahara et al., Phys. Rev. Lett. 97, 146803 (2006).

[8] T. Hirahara et al., New J. Phys. 10, 083038 (2008).

[9] P. Hofmann, Prog. Surf. Sci. 81, 191 (2006).

[10] P. Hofmann et al., Phys. Rev. B 71, 195413 (2005).

[11] K. Sugawara et al., Phys. Rev. Lett. 96, 046411 (2006).

[12] C. R. Ast et al., Phys. Rev. Lett. 98, 186807 (2007).

[13] D. Pacilé et al., Phys. Rev. B 73, 245429 (2006).

[14] L. Moreschini et al., Phys. Rev. B 80, 035438 (2009).

[15] C. R. Ast et al., Phys. Rev. B 75, 201401(R) (2007).

[16] G. Bihlmayer, S. Blügel, and E. V. Chulkov, Phys. Rev. B 75, 195414 (2007).

[17] H. Mirhosseini et al., Phys. Rev. B 79, 245428 (2009).

[18] C. R. Ast et al., Phys. Rev. B 77, 081407(R) (2008).

[19] F. Meier et al., Phys. Rev. B 79, 241408(R) (2009).

[20] T. C. Chiang, Surf. Sci. Rep. 39, 181 (2000).

[21] M. M. Oezer et al., Science 316, 1594 (2007).

[22] L. Aballe et al., Phys. Rev. Lett. 93, 196103 (2004).

[23] S. Mathias et al., Phys. Rev. Lett. 97, 236809 (2006).

[24] S. Mathias et al., Phys. Rev. Lett. 103, 026802 (2009).

[25] T. Hirahara et al., Phys. Rev. B 76, 153305 (2007).

[26] T. Hirahara et al., Phys. Rev. B 75, 035422 (2007).

[27] J. Hugo Dil et al., Phys. Rev. Lett. 101, 266802 (2008).

[28] L. Petersen et al., Surf. Sci. 459, 49 (2000).

[29] For a program description, see http://www.flapw.de.

[30] D. Kaminski et al., Surf. Sci. 575, 233 (2005).

[31] C. Li et al., Phys. Rev. B 42, 5433 (1990).

[32] J. Nitta et al., Phys. Rev. Lett. 78, 1335 (1997).

[33] J. Premper et al., Phys. Rev. B 76, 073310 (2007).

[34] J. I. Pascual, et al., Phys. Rev. Lett. 93, 196802 (2004).

[35] I. Gierz et al., Phys. Rev. Lett. 103, 046803 (2009). 